\title{
EFEITO DAS CONDIÇÕES PRÉ-ABATE SOBRE A QUALIDADE DA CARNE DE SUÍNOS PESADOS
}

\author{
EFFECT OF PRE-SLAUGHTER CONDITIONS ON MEAT QUALITY \\ OFHEAVY-WEIGHTPIGS
}

\author{
Dalla Costa, O.A. ${ }^{1 *}$, Ludke, J.V. ${ }^{1}$, Costa, M.J.R.P. ${ }^{2}$, Faucitano, L. ${ }^{3}$, Peloso, J.V. ${ }^{4}$ \\ e Dalla Roza, D. ${ }^{5}$
}

\begin{abstract}
${ }^{1}$ Embrapa Suínos e Aves. Caixa Postal 21. CEP 89700-000. Concórdia-Santa Catarina. Brasil. *osmar@cnpsa.embrapa.br

${ }^{2}$ Universidade Estadual Paulista. FCAV/UNESP. Jaboticabal-São Paulo. Brasil.

${ }^{3}$ Agriculture and Agri-Food Canada, Dairy and Swine Research and Development Centre. LennoxvilleQuebec. Canadá.

${ }^{4}$ Sadia S.A. Concórdia-Santa Catarina. Brasil.

${ }^{5}$ Triel-HT Indústria de Equipamentos Rodoviários Ltda. Erechim-Rio Grande do Sul. Brasil.
\end{abstract}

\section{PalaVRas ChaVe ADICIONAIS}

Carroceria em piso duplo. Manejo pré-abate. Perda por gotejamento.

\section{RESUMO}

O objetivo da pesquisa foi avaliar o tempo de jejum (FT= 9, 12, 15 e 18 horas) dos suínos na granja e a condição de transporte representado pela posição do suíno na carroceria do caminhão sobre a qualidade de carne avaliada através da perda de líquido por gotejamento (DLL), $\mathrm{pH}_{1}$ mensurado aos 45 minutos após o abate e $\mathrm{pH}_{u}$ mensurado 24 horas após o abate. Foram utilizadas 192 fêmeas com peso vivo médio de 133ะ11 $\mathrm{kg}$, oriundas de duas granjas. As posições na carroceria avaliadas foram frente, meio e atrás (TCL) e piso inferior e superior (LUD). No modelo estatístico foram considerados os efeitos de bloco (BL= granja), FT, TCL e LUD e a interação (BL xFT). $\mathrm{O}$ FT teve influência sobre $0 \mathrm{pH}_{1}$ do músculo Longissimus dorsi e sobre o $\mathrm{pH}_{u}$ dos músculos Semispinalis capitis (SC), Longissimus dorsi (LD) e Semimembranosus (SM). Tempo de jejum inferior ou igual a 12 horas resultou em valores de $\mathrm{pH}_{u}$ menores nos músculos LD e $\mathrm{SM}$ e jejum superior a 15 horas determinou maior $\mathrm{pH}_{u}$ no $\mathrm{SC}$. Não houve efeito $(p>0,05)$ dos fatores de variação avaliados sobre a DLL. A TCL influenciou significativamente o $\mathrm{pH}_{1}$ dos músculos SC, LD e $\mathrm{SM}$ e o $\mathrm{pH}_{4}$ dos músculos LD e SM. Suínos transportados no meio ou na posição posterior da

Recibido: 21-05-08. Aceptado: 16-12-08.

\section{AdDitional KEYWORDS}

Double deck lorry. Pre slaughter management. Drip loss.

carroceria não apresentaram diferença no $\mathrm{pH}_{1}$ nos músculos avaliados. Porém, suínos transportados no meio da carroceria apresentaram maior $\mathrm{pH}_{u}$ no LD e SM. Conclui-se que o tempo de jejum nas granjas deve ser de 15 horas para a obtenção de maior freqüência de $\mathrm{pH}_{u}$ nos músculos avaliados na faixa de $\mathrm{pH}$ normal $\left(5,55<\mathrm{pH}_{\mathrm{u}} \leq 5,90\right)$ e que no transporte de curta duração a posição dos suínos na carrocería afeta o $\mathrm{pH}$ da carne, no entanto, sem afetar a sua qualidade.

\section{SUMMARY}

The objective of this study was to evaluate pig fasting time at the farm ( $\mathrm{FT}=9,12,15$ or 18 hours) and pig position into the pig lorry compartment on pork quality through liquid drip loss (DLL), $\mathrm{pH}_{1}$ evaluated at 45 minutes post slaughter and $\mathrm{pH}_{4}$ evaluated 24 hours post slaughter. One hundred ninety two females, weighing $133 \pm 11 \mathrm{~kg}$, from two farms were used. Pig locations were evaluated on truck compartment considering front, middle and rear (TCL) position and top or botton decks (LUD). The following effects were considered in the statistical model: block $(B L=$ farm), FT, TCL, LUD and the interaction between $\mathrm{BL}$ and FT. The FT 
DALLA COSTA, LUDKE, COSTA, FAUCITANO, PELOSO E DALLA ROZA

affected significantly the $\mathrm{pH}_{1}$ of Longissimus dorsi (LD) muscle, and $\mathrm{pH}_{\mathrm{u}}$ of Semispinalis capitis (SC), Longissimus dorsi (LD) and Semimembranosus (SM) muscles. Fasting time at farm equal or shorter than 12 hours resulted in carcasses with lower $\mathrm{pH}_{\mathrm{u}}$ values at $\mathrm{LD}$ and $\mathrm{SM}$ muscles and fasting time above 15 hours resulted in higher $\mathrm{pH}_{u}$ on SC. There were no observed effect $(p>0.05)$ of the evaluated sources of variation on DLL. TCL affected $\mathrm{pH}_{1}$ of SC, LD and SM muscles, and $\mathrm{pH}_{u}$ of LD and SM muscles. Pigs located in middle or rear position in the lorry had no difference in $\mathrm{pH}_{1}$ of the evaluated muscles. But pigs located in the middle position of the lorry had greater values of $\mathrm{pH}_{u}$ on LD and SM. It is stated that pigs fasting time at farm need to be close to 15 hours in aim of obtain higher frequency of $\mathrm{pH}_{\mathrm{u}}$ values in the normal range $\left(5.55<\mathrm{pH}_{\mathrm{u}} \leq 5.90\right)$ and that in short time transport the pigs location in lorry's compartment had effect on meat $\mathrm{pH}$, however, without affect its quality.

\section{INTRODUÇÃO}

No manejo pré-abate dos suínos, o jejum (Dalla Costa et al., 2008a), a mistura de lotes (Faucitano, 2007), o tempo de descanso no frigorífico (Aaslyng e Barton Gade, 2001) e melhoria do manejo no pré-abate (Hambrecht, 2004; Carr et al., 2008) são fatores essenciais para manutenção da qualidade das carcaças e da carne. Para avaliar qualidade de carne existem parâmetros que têm alta representatividade, baixo custo e reduzida complexidade entre os quais estão o pH aos 45 minutos após o abate $\left(\mathrm{pH}_{1}\right)$ e $\mathrm{pH}$ 24 horas após o abate $\left(\mathrm{pH}_{\mathrm{u}}\right)$ e a perda de líquido por gotejamento (Barton Gade et al., 1996).

O jejum pré-abate nos suínos é relevante para o produtor e para o abatedouro, pois contribui para a economía de ração, redução da taxa de mortalidade durante o transporte, aumento da velocidade e facilidade no processo de evisceração, redução no potencial de contaminação, redução do volume de dejetos e melhoria na padronização do rendimento de carcaça e manutenção da qualidade da carne (Dalla Costa et al., 2008b). O tempo de jejum dos suínos na granja recomendado é de 10 a 24 horas
(Murray, 2000), porém tem variado de acordo com o país e com o perfil genético dos suínos. Na França recomenda-se um jejum entre 12 e 18 horas antes do carregamento e não mais do que 24 horas de jejum total (Chevillon, 1994). Guardia et al. (1996) recomendaram um jejum de 12 a 18 horas, enquanto que Eikelenboon et al. (1991) recomendaram entre 16 e 24 horas. Segundo Magras et al. (2000), quando o objetivo é obter maior porcentagem de suínos que apresentem peso de estomago menor que $1,4 \mathrm{~kg}$, deve ser utilizado um período total de jejum entre 22 a 28 horas.

Duas das principais características no transporte de suínos ao abate correspondem à distância percorrida e o tempo decorrido entre o carregamento na granja e o alojamento nas baias de descanso no abatedouro. Christensen et al. (1994) relataram que em sete países europeus a maioria dos suínos destinados ao abate eram transportados a distância menor do que $100 \mathrm{~km}$. Ryan (2007) cita que nos EUA metade dos suínos abatidos são transportados a distâncias inferiores a $161 \mathrm{~km}$. Na região Sul do Brasil estima-se que a distância média para o transporte de suínos até o abatedouro seja em torno de $80 \mathrm{~km}$ com duração não superior a três horas. Inúmeras pesquisas indicam que suínos submetidos a transporte de curta duração apresentam ao final da jornada síntomas de estresse e efeitos sobre a qualidade da carne, decorrentes de manejo, mais pronunciados do que os submetidos a viagens de média ou longa duração. Isto se deve ao ritmo de adaptação dos suínos às situações de estresse.

Durante o transporte, considerando carrocerías bem dimensionadas e sob taxa de lotação adequada existem duas condições de estresse de ordem física que são importantes: o microclima interno (como resultado da temperatura, umidade relativa do ar e taxa de ventilação) e a intensidade de trepidação durante o movimento gerado pela interação entre a condição intrínseca do veículo, a forma de deslocamento, o tipo de

Archivos de zootecnia vol. 59, núm. 227, p. 392. 
suspensão instalada no veículo e as condições das estradas (SCAHAW, 1999). A posição do box dentro da carroceria é uma fonte de variação que pode influenciar o bem-estar dos animais e a qualidade da carne do suínos (Broom et al., 2002). Segundo Dalla Costa et al. (2007a , b) isso ocorre porque os animais transportados na posição anterior da carroceria estão expostos a uma menor taxa efetiva de ventilação e podem estar submetidos a temperaturas mais altas, enquanto que os suínos transportados na posição posterior da carroceria estão sujeitos aos fenômenos estressores decorrentes da vibração e transferência intensa e direta dos impactos transmitidos pelos eixos de sustentação do rodado. Neste contexto, em carrocerías abertas, espera-se um efeito das condições ambientais quando os suínos de abate são transportados por períodos significativos sob condições climáticas extremas.

Estudos realizados por Barton Gade et al. (1996) em condições características dos países escandinavos, indicaram ausência de efeito do compartimento (frente, intermediário e posterior) e do piso (inferior ou superior) da carroceria sobre o $\mathrm{pH}_{1}$ do músculo Semimembranosus, porém demonstraram um efeito significativo do compartimento sobre o $\mathrm{pH}_{u}$ dos músculos Biceps femoris e Semimembranosus e efeito de interação entre os compartimentos da carroceria e o piso sobre o $\mathrm{pH}_{u}$ dos músculos Semispinalis capitis e Longissimus dorsi.

Nesta perspectiva, o objetivo do trabalho foi avaliar os efeitos do tempo de jejum na granja e da localização dos suínos dentro de uma carrocería com dois pisos sobre a qualidade da carne quando animais de abate pesados são transportados a curtas distâncias no verão ou inverno.

\section{MATERIAL E MÉTODOS}

O experimento foi realizado em duas épocas do ano, sob condições normais de produção, em duas granjas comerciais de terminação de suínos localizadas em Concórdia, Santa Catarina, Brasil. Foram utilizados animais de mesma origem genética, selecionada para ausência do gen Halotano, e oriundos de cruzamentos industriais para abate. Nas diferentes operações durante o período pré-abate os animais foram conduzidos segundo um programa de manejo préabate racional com pessoas treinadas, sem a utilização de choques elétricos e em condições padronizadas de embarque e desembarque dos suínos.

No manejo pré-abate, os suínos foram submetidos a tempos de jejum de 9, 12, 15 ou 18 horas antes do carregamento. O sistema de alimentação adotado nas granjas era o de fornecimento controlado de ração três vezes ao dia (de manhã, ao meio dia e ao anoitecer). A adoção dos diferentes tempos de jejum pré-embarque possibilitou que o último fornecimento de ração fosse às 10:00; 13:00; 16:00 e 19:00 horas para os tempos de jejum de 18, 15, 12 e 9 horas, respectivamente. As granjas avaliadas apresentavam capacidade para terminação de 500 suínos em sistema all in-all out. Em cada uma delas foram escolhidas aleatoriamente 4 baias por tempo de jejum, em cada baia foram utilizadas seis fêmeas, totalizando 96 suínos em 16 baias. Ao total para a realização do experimento foram utilizadas 192 fêmeas com peso médio de abate de $133 \pm 11 \mathrm{~kg}$. Em todas as etapas do manejo pré-abate (carregamento, transporte, descarga, descanso no abatedouro e abate) os animais foram manejados em grupos de seis mantendo a composição original das baias de origem.

Os animais foram transportados em um modelo de carroceria metálica monobloco dupla, isto é, com piso em dois andares conforme apresentado na figura $\mathbf{1}$, com capacidade de transporte de 96 suínos de abate, representando uma densidade média de $0,45 \mathrm{~m}^{2} /$ suíno. O modelo de carroceria utilizado é construído em aço estrutural, tubos galvanizados, assoalho em alumínio xadrez, frente totalmente fechada com con- 


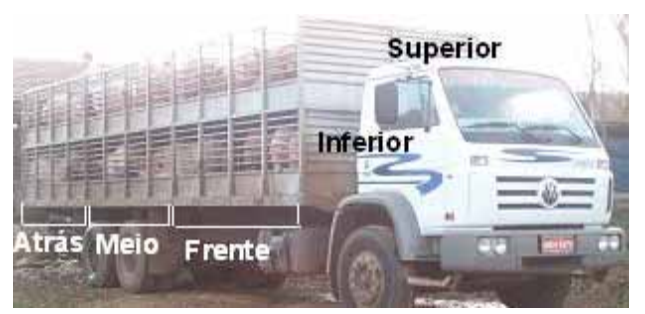

Figura I. Modelo de carroceria utilizada nas avaliações. (Model of lorry used at the evaluations).

trole de ventilação modelo PVC, com área total de 43,2 $\mathrm{m}^{2}$, sendo $9 \mathrm{~m}$ de comprimento 2,4 m de largura $0,90 \mathrm{~m}$ de altura fixa, onde foram transportados 96 suínos em 16 boxes cada qual com dimensão de $2,7 \mathrm{~m}^{2}$ (com oito boxes por piso e quatro em cada lateral), com portões internos de girar e portão traseiro de girar duplo, com sistema de abertura da carroceria tipo guilhotina central e provido de rampa móvel de metal de $3 \mathrm{~m}$ de comprimento para embarque e desembarque. Para a avaliação da posição dos boxes dentro da carroceria foi considerado como posição anterior (box da frente) os localizados nas laterais esquerda e direita logo após a cabine do caminhão, como posição intermediária (box do meio) os $2^{\circ}$ e $3^{\circ}$ box localizados nas laterais esquerda e direita e, posição posterior (box de trás) os últimos localizados na parte traseira da carrocería. Na tabela I estão apresentadas as condições em que as avaliações foram realizadas.

Nas duas avaliações realizadas o horario de carregamento (04:00 horas da manhã), o veículo usado no transporte, o condutor do veículo e o local de abate foram mantidos padronizados. Os animais oriundos das duas granjas foram submetidos ao mesmo manejo durante o transporte, descarga, período de descanso e foram alojados nas mesmas baias de descanso no abatedouro. Os abates foram realizados no período da manhã observando o tempo padrão de três horas de descanso nas baias do abatedouro onde os animais tinham acesso à água por meio de bebedouros do tipo chupeta. Foram aplicadas iguais condições de abate e manejo das carcaças. A insensibilização via eletronarcose foi aplicada automaticamente, transferindo alta voltagem $(700 \mathrm{~V})$ e amperagem acima de 1,25 Amps (Valhalla, Stork RMS b.v., Lichtenvoorde, Holanda). Após a insensibilização, os animais eram imediatamente sangrados na posição horizontal e ao fim da mesa de sangria suspensos em nora contínua na linha de abate.

Após a conclusão dos procedimentos de abate as carcaças permaneceram em câmara fria submetidas a temperaturas variando entre $1^{\circ} \mathrm{C}$ e $4^{\circ} \mathrm{C}$ por 24 horas. As medidas de $\mathrm{pH}$ foram realizadas na meia carcaça esquerda nos músculos Semispinalis capitis (SC), Longissimus dorsi (LD) e Semimembranosus (SM), 45 minutos ( $\mathrm{pH}_{1}$ ) e 24 horas após o abate $\left(\mathrm{pH}_{4}\right)$. Na avaliação do $\mathrm{pH}$, nos dois abates, foi utilizado o mesmo pHmetro portátil com eletrodo DXK-S7/25 protegido para inserção no músculo. $\mathrm{O}_{\mathrm{pH}}$ foi medido antes das carcaças serem dispostas na câmara fría. Para a realização da medida do $\mathrm{pH}_{u}$ as carcaças foram retiradas da cámara e dispostas brevemente à temperatura ambiente.

A porcentagem de perda de água por gotejamento (DLL) do músculo Semimembranosus foi determinada em 50\% das carcaças dos animais que participaram deste estudo. Vinte e quatro horas após o abate foram coletadas amostras de aproximadamente $110 \mathrm{~g}$ de músculo, livre de gordura, que após a pesagem, foram suspensas em redes de nylon seladas dentro de sacos plásticos, e assim permaneceram na câmara fria submetidos a temperaturas variando entre $1^{\circ} \mathrm{C} \mathrm{e} 4^{\circ} \mathrm{C}$ por 24 horas. A avaliação foi realizada segundo os procedimentos descritos por Honikel (1998). A porcentagem de DLL foi calculada como a diferença entre o peso inicial e o peso final da amostra, cujo resultado foi dividido pelo peso inicial e multiplicado por 100 .

Na análise das variáveis foi utilizado o método de quadrados mínimos, com a

Archivos de zootecnia vol. 59, núm. 227, p. 394. 
Tabela I. Descrição das condições experimentais observadas nas avaliações realizadas. (Description of observed experimental conditions on performed evaluations).

\begin{tabular}{|c|c|c|}
\hline $\begin{array}{l}\text { Condições experimentais } \\
\text { Estação e mês do ano }\end{array}$ & $\begin{array}{l}\text { Avaliação } 1 \\
\text { Inverno/junho }\end{array}$ & $\begin{array}{c}\text { Avaliação } 2 \\
\text { Verão/fevereiro }\end{array}$ \\
\hline $\mathrm{N}^{\circ}$ de baias por tratamento e por granja & $4 / 16$ & $4 / 16$ \\
\hline $\mathrm{N}^{\circ}$ de animais por tratamento e avaliação & $24 / 96$ & $24 / 96$ \\
\hline Peso médio, kg & $134 \pm 10$ & $132 \pm 12$ \\
\hline $\mathrm{kg}$ de suíno vivo por $\mathrm{m}^{2}$ de carrocería & $298 \pm 22$ & $293 \pm 27$ \\
\hline \multicolumn{3}{|l|}{ Temperaturas na granja durante 24 horas } \\
\hline Mínima absoluta, ${ }^{\circ} \mathrm{C}$ & 0,0 & 13,0 \\
\hline Máxima absoluta, ${ }^{\circ} \mathrm{C}$ & 29,0 & 33,5 \\
\hline Média das mínimas, ${ }^{\circ} \mathrm{C}$ & 12,8 & 19,9 \\
\hline Média das máximas, ${ }^{\circ} \mathrm{C}$ & 18,8 & 27,2 \\
\hline Média geral, ${ }^{\circ} \mathrm{C}$ & 15,3 & 24,3 \\
\hline Umidade relativa do ar, \% & $74,8 \pm 6,2$ & $69,1 \pm 8,6$ \\
\hline Distância da granja ao abatedouro, km & 23 & 45 \\
\hline Tempo para carregamento, minutos & 29 & 45 \\
\hline \multicolumn{3}{|l|}{ Durante o transporte } \\
\hline Tempo de jornada, minutos & 31 & 75 \\
\hline Temperatura média, ${ }^{\circ} \mathrm{C}$ & 13,5 & 22,8 \\
\hline Média da umidade relativa do ar, \% & 91,3 & 82,0 \\
\hline \multicolumn{3}{|l|}{ Nas baias de descanso do abatedouro } \\
\hline Temperatura média, ${ }^{\circ} \mathrm{C}$ & 12,2 & 24,8 \\
\hline Média da umidade relativa do ar, \% & 95,5 & 75,3 \\
\hline
\end{tabular}

aplicação do procedimento GLM do programa Statistical Analysis System (SAS, 2001) com o seguinte modelo matemático :

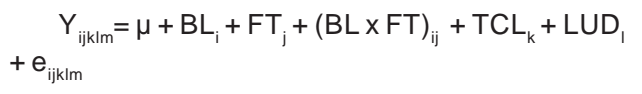

sendo:

$\mathrm{Y}_{\mathrm{ijk} / \mathrm{m}}=\mathrm{pH}_{1}, \mathrm{pH}_{\mathrm{u}}$ e DLL;

$\mu=$ média geral;

$\mathrm{BL}_{\mathrm{i}}=$ bloco (estação do ano ou granja, $\mathrm{i}=1$ inverno e 2 verão);

$\mathrm{FT}_{\mathrm{j}}=$ tempo de jejum dos suínos na granja antes do carregamento, $\mathrm{j}=1$, 2, 3, 4 (tempo de jejum de $9,12,15,18$ horas, respectivamente);

$(B L \times F T)_{i j}$ interação entre bloco e tempo de jejum dos suínos na granja antes do carregamento;

$\mathrm{TCL}_{\mathrm{k}}=$ posição do animal na carroceria, $\mathrm{k}=1$ (frente - primeiro box da carroceiria), 2 (meio - os dois box intermediários da carroceria) e 3 (atrás o último box da carroceria);

$L_{U}=$ piso da carroceria, $\mathrm{I}=1$ (inferior) e 2 (superior);

$\mathrm{e}_{\mathrm{ijklm}}=$ erro aleatório. Quando necessário foi utilizado o teste de médias t a $5 \%$ de probabilidade.
Exceto a interação BLXFT nenhuma outra interação dupla ou tripla foi incluída no modelo e isto é decorrente da estrutura expeimental adotada.

\section{RESULTADOSEDISCUSSÃO}

Nas avaliações realizadas foi considerado o maior grau de suscetibilidade ao estresse utilizando fêmeas suínas pesadas para caracterizar o potencial máximo da influência das condições de manejo pré-abate em estudo. A caracterização da carrocería usada nas avaliações indica duas condições importantes. Segundo Broom et al. (2002) deve ser considerado um padrão de área útil $\left(\mathrm{A}, \mathrm{m}^{2}\right)$ por suíno transportado a curtas distâncias que segue a equação:

$$
A=0,0192 W^{0,67}
$$

onde:

W é o peso médio do suíno em kg.

Archivos de zootecnia vol. 59, núm. 227, p. 395. 
Nestas condições a área média disponível nas presentes avaliações deveria ser de 0,5085 m² por suíno, ou seja, uma área cerca de $13 \%$ maior. A altura característica apresentada em cada piso na carrocería é de $90 \mathrm{~cm}$ e é adequada para animais com peso vivo de até $100 \mathrm{~kg}$. Porém, conforme apresentado na tabela II, a temperatura média e a umidade relativa do ar observadas no interior da carroceria durante as etapas do transporte estão de acordo com a recomendação defendida por SCAHAW (1999).

Na análise estatística não foram verificados efeitos significativos ( $\mathrm{p}>0$ 0,05) para época do ano $(\mathrm{BL}=$ granja) para a perda de líquido por gotejamento, $\mathrm{pH}_{1}$ e $\mathrm{pH}_{\text {. }}$. E, também não foram verificados efeitos de interação $(p>0,05)$ entre o tempo de jejum na granja e época do ano para os parâmetros avaliados. Isto significa que as respostas para o tempo de jejum na granja estavam independentes da época do ano (inverno ou verão). No acompanhamento das temperaturas durante 24 horas antes do carregamento dos

Tabela II. Média das temperaturas observadas durante as etapas de transporte e por local de avaliação.(Mean temperatures observed during transport stages and by evaluation point).

\begin{tabular}{lcc}
\hline $\begin{array}{l}\text { Etapas do transporte } \\
\text { ou local de avaliação }\end{array}$ & $\begin{array}{c}\text { Temperatura } \\
{ }^{\circ} \mathrm{C}\end{array}$ & $\begin{array}{c}\text { URA } \\
\%\end{array}$ \\
\hline $\begin{array}{l}\text { Carregamento dos suínos } \\
\text { Caminhão parado granja }\end{array}$ & $\begin{array}{c}17,1 \pm 0,5 \\
18,7 \pm 0,5\end{array}$ & $89,3 \pm 0,8$ \\
Durante o transporte & $17,8 \pm 0,5$ & $85,8 \pm 0,9$ \\
Box frente & $18,5 \pm 0,4$ & $88,7 \pm 0,7$ \\
Box meio & $18,7 \pm 0,4$ & $83,0 \pm 0,6$ \\
Box atrás & $17,2 \pm 0,4$ & $88,2 \pm 0,6$ \\
Piso inferior & $18,6 \pm 0,3$ & $86,1 \pm 0,5$ \\
Piso superior & $18,7 \pm 0,3$ & $87,3 \pm 0,5$ \\
Caminhão parado no & & \\
abatedouro & $19,1 \pm 0,5$ & $83,7 \pm 0,8$ \\
\hline
\end{tabular}

Não houve $(p>0,05)$ efeito sobre a temperatura e a umidade relativa do ar (URA). animais, foi possível verificar que ocorreram baixas e altas temperaturas no inverno e no verão, respectivamente, porém no dia do abate (no transporte e nas baias de descanso no abatedouro) as temperaturas observadas se situaram muito próximas à média geral observada no dia anterior ao abate, tanto para o inverno quanto para o verão. Os resultados obtidos no presente experimento diferem dos descritos por Carr et al. (2008).

A perda de líquido por gotejamento em 48 horas com um valor médio de $5,66 \pm 1,84 \%$ não foi afetada pela posição $(p=0,6749)$ dos animais na carroceria do caminhão (frente, meio ou posterior), pela sua localização ( $\mathrm{p}=$ 0,7849 ) no piso (inferior ou superior) e pelo tempo de jejum na granja $(p=0,1747)$ conforme apresentado na figura 2 . O valor médio para o DLL acima de 5,0\% indica potencialmente presença de carne PSE (Otto et al., 2004). Esses resultados estão de acordo com os encontrados por Murray e Jones (1994) e Murray et al. (2001). Porém, diferem dos encontrados por Jones et al. (1985) e Eikelenboom et al. (1991) que observaram que os suínos submetidos a jejum de 24 a 48 horas apresentaram valores menores de perda de líquido por gotejamento no músculo Longissimus dorsi $(\mathrm{p}<0,05)$ em comparação aos animais que não receberam jejum na granja. Valores de DLL abaixo de 2,0\% estão associados com características de carne DFD enquanto que os valores de DLL acima de 5,0\% estão associados com características de carne PSE (Ryan, 2007).

O posicionamento dos suínos na carrocería (frente, meio e posterior) influenciou o $\mathrm{pH}_{1}$ dos músculos Semispinalis capitis $(p=0,0406)$, Longissimus dorsi $(p=0,0334)$ e Semimembranosus $(\mathrm{p}=0,0299)$, também influenciou o $\mathrm{pH}_{u}$ dos músculos Longissimus dorsi $(\mathrm{p}=0,0020)$ e Semimembranosus ( $\mathrm{p}=0,0004)$ porém, não influenciou o $\mathrm{pH}$ do músculo Semispinalis capitis $(\mathrm{p}=$ 0,0633 ) conforme apresentado na figura 3 . Os suínos transportados nos box do meio e parte posterior da carroceria apresentaram

Archivos de zootecnia vol. 59, núm. 227, p. 396. 


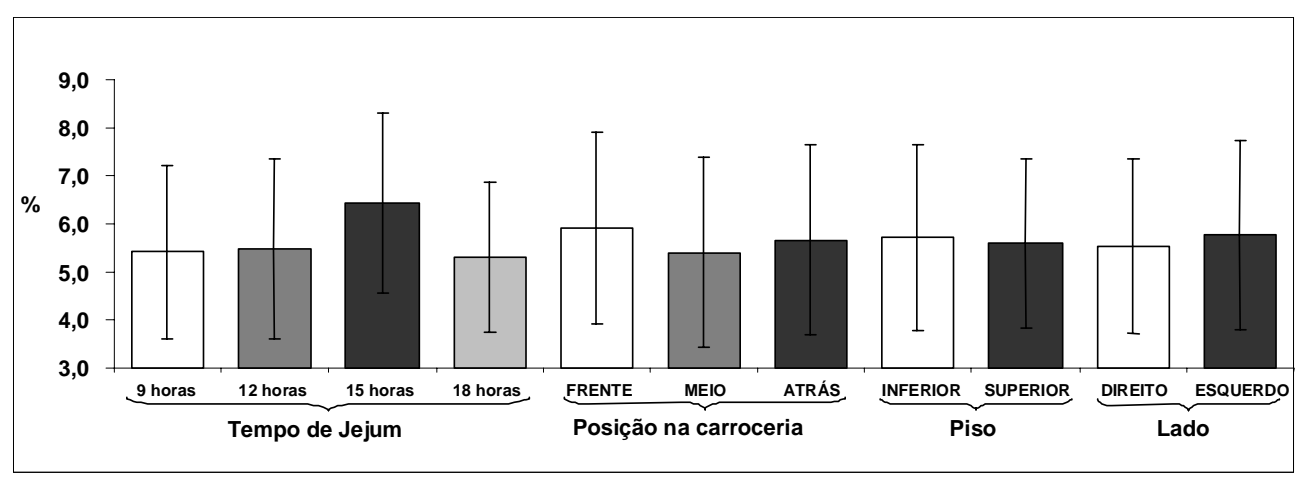

Figura 2. Médias ajustadas e desvios-padrão da porcentagem de perda de água por gotejamento no músculo Semimembranosus (SM) como efeito do tempo de jejum dos suínos na granja e posição do box dentro da carroceria do caminhão. (Mean and standard deviation of drip loss percentage on Semimembranosus muscle (SM) as effect of swine fasting time at farm and position in truck lorry).

valores de $\mathrm{pH}_{1}$ que não diferiram entre si ( $\mathrm{p}>0,05)$ para os três músculos avaliados. Nos suínos transportados no meio da carroceria foram medidos maiores valores de $\mathrm{pH}_{\mathrm{u}}$ dos músculos Longissimus dorsi e Semimembranosus em relação aos transportados na posição anterior e posterior da carroceria. Esses resultados diferem dos encontrados por Barton Gade et al. (1996) que não constataram efeito da posição na carroceria sobre o $\mathrm{pH}_{1}$ no músculo de $\mathrm{SC}$, e do $\mathrm{pH}_{u}$ dos músculos Bíceps femoris (BF) e $\mathrm{SC}$, bem como efeito da interação entre o piso e posição na carroceria sobre o $\mathrm{pH}_{1}$ de $\mathrm{LD} \mathrm{e} \mathrm{pH}_{u}$ de LD e SC. Não foram observadas diferenças significativas condicionadas pelo piso (inferior e superior) sobre os valores de pH medidos nos músculos. Assim, os valores de $\mathrm{pH}_{1}$ dos músculos $\mathrm{SC}(\mathrm{p}=0,9372), \mathrm{LD}$ $(p=0,6969)$ e SM $(p=0,4635)$ e do $\mathrm{pH}$ dos músculos SC ( $p=0,2291), \operatorname{LD}(p=0,3689$ ) e $\operatorname{SM}(p=0,3849)$ não sofreram influência deste fator de variação considerado. Esses resultados estão de acordo com os obtidos por Barton Gade et al. (1996) que não observaram efeito do piso (inferior ou superior) da carroceria sobre o $\mathrm{pH}_{1}$ de $\mathrm{SM}$, porém determinaram um efeito significativo do compartimento sobre o $\mathrm{pH}_{u}$ de BF e SM e da interação entre os compartimentos da carroceria e piso sobre o $\mathrm{pH}_{\mathrm{u}}$ dos músculos SC e LD. Nas condições de transporte verificadas (distância, duração da viagem, qualidade da estrada, características intrínsecas do veículo e clima) a posição dos suínos na carroceria considerando os parâmetros porcentagem de perda de água e o pH não acarretou efeito negativo sobre a qualidade da carne. Embora diferenças estatísticas significativas tenham sido observadas para o $\mathrm{pH}_{\mathrm{H}}$ estas ocorreram dentro da amplitude de $\mathrm{pH}$ entre 5,55 e 5,90 considerada a faixa de $\mathrm{pH}$ adequada carne normal com ausência de PSE e DFD.

Segundo Faucitano (2007) o tempo de jejum total durante o manejo pré-abate está associado a logística dos abatedouros porém deve se situar dentro de limites previamente definidos pois a sua duração compromete o bem-estar, a perda de peso da carcaça e a qualidade da carne do suínos. O tempo de jejum dos suínos na granja não influenciou significativamente $\mathrm{o} \mathrm{pH}_{1}$ nos músculos SC $(p=0,0815)$ e SM $(p=0,1630)$. Contudo, houve efeito significativo $(\mathrm{p}=$ 0,0401) no $\mathrm{pH}_{1}$ de LD e no $\mathrm{pH}_{u}$ dos três músculos avaliados $(p=0,0001)$ conforme apresentado na figura 4 . Os suínos que 

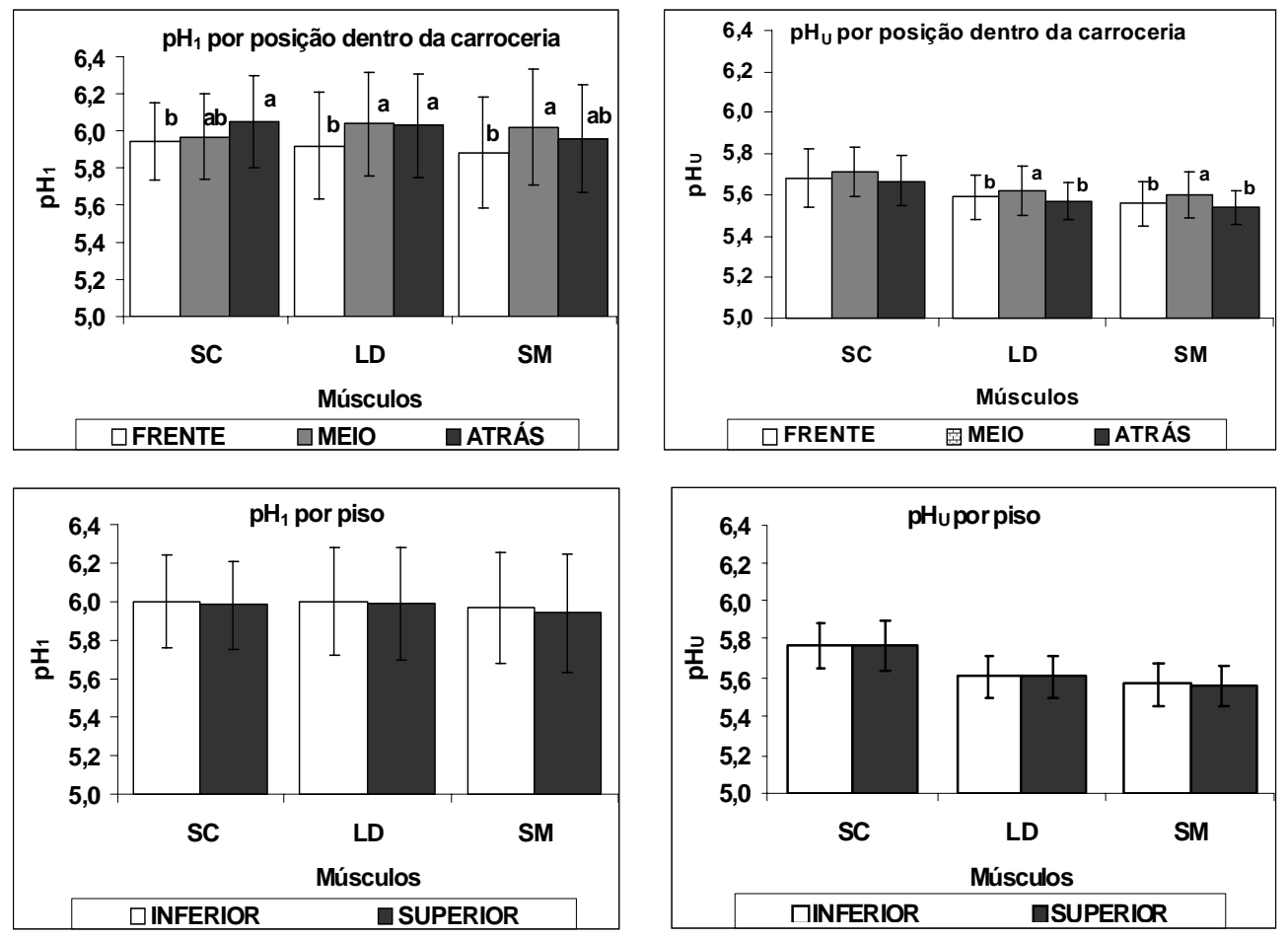

Médias seguidas de letras minúsculas distintas diferem significativamente pelo teste $t(p<0,05)$.

Figura 3. Médias ajustadas de $\mathrm{pH}_{\text {e }} \mathrm{pH}$ no músculo Semispinalis capitis (SC), Longissimus dorsi (LD) e Semimembranosus (SM), por posição do box dentro da carroceria do caminhão. (Means of $\mathrm{pH}_{1}$ and $\mathrm{pH}_{\mathrm{u}}$ on Semispinalis capitis (SC), Longissimus dorsi (LD) and Semimembranosus (SM) muscles as affected by pig location in truck lorry).

receberam jejum de 15 horas na granja apresentaram maiores valores do $\mathrm{pH}_{1}$ no $\mathrm{LD}$, porém não diferiram $(\mathrm{p}=0,0752$ e $\mathrm{p}=10,1570$ ) dos animais que receberam jejum de 9 e 18 horas. Aqueles que receberam jejum de 12 horas apresentaram menores valores de $\mathrm{pH}_{1}$, e não diferiram dos jejuns de 9 e 18 horas. Suínos submetidos a um jejum de 12 horas na granja apresentaram menores valores $\mathrm{pH}_{u}$ nos músculos SC e LD. Os animais que receberam jejum de 18 horas apresentaram maiores valores de $\mathrm{pH}_{u}$ no SC e suínos submetidos a jejum de 15 e 18 horas apresentaram maiores valores de $\mathrm{pH}_{\mathrm{u}}$ nos músculo LD e SM $(\mathrm{p}<0,05)$. O manejo préabate utilizado no presente trabalho (jejum, duração do transporte e descanso no abatedouro) difere dos procedimentos empregados no manejo pré-abate dos suínos tais como tempo de jejum na granja (Magras et al., 2000; Murray, 2000) e período de descanso no frigorífico (Aaslyng e Barton Gade, 2001). Os resultados obtidos no presente estudo validam os encontrados na literatura (Eikelenboom et al., 1991; Murray e Jones, 1994; Wittmann et al., 1994) que verificaram efeito de diferentes tempos de jejum ( 0 a 72 horas) na granja sobre o $\mathrm{pH}$ dos músculos. Contudo, diferem dos resultados constatados por Smet et al. (1996) e Murray et al. (2001), que avaliaram o efeito do jejum (0 e 24; 0 e 15 horas, respectivamente) e não

Archivos de zootecnia vol. 59, núm. 227, p. 398. 

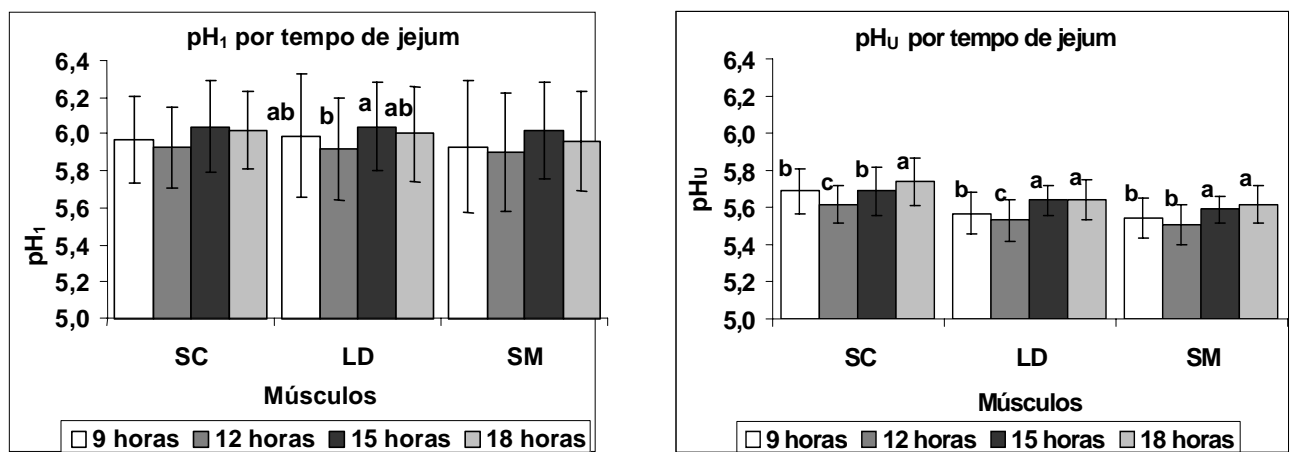

Médias seguidas de letras minúsculas distintas diferem significativamente pelo teste $t(p<0,05)$.

Figura 4. Médias ajustadas do $\mathrm{pH}_{\mathrm{f}}$ e $\mathrm{pH}_{u}$ nos músculos Semmispinalis capitis(SC), Longissimus dorsi $(L D)$ e Semimembranosus (SM), por tempo de jejum dos suínos na granja. (Means of $\mathrm{pH}_{1}$ and $\mathrm{pH}_{u}$ on Semmispinalis capitis (SC), Longissimus dorsi (LD) and Semimembranosus (SM) muscles as affected by pig fasting time at farm).

verificaram efeito do jejum dos suínos sobre os $\mathrm{pH}_{1}$ e $\mathrm{pH}_{u}$ do músculo LD.

Uma alta porcentagem de carcaças com $\mathrm{pH}_{\mathrm{u}}$ elevado (maior que 5,90) nos músculos SC, LD e SM, pode ser um indicador de carnes com características de DFD. Conforme apresentado na tabela III, nas 192 carcaças avaliadas foi caracterizado que apenas $4,2 \%, 1,1 \%$ e $0 \%$ (respectivamente, para os músculos SC, LD e SM) apresentaram características DFD. Porém, verificouse uma alta porcentagem de carcaças com $\mathrm{pH}_{u}$ baixo (menor ou igual a 5,55) nos músculos LD e SM (36,7\% e 39,3\%, respectivamente), o que indica carcaças com característica de PSE. Somente 62,3\% e 60,7\% das carcaças avaliadas apresentaram $\mathrm{pH}_{u}$ dos músculos LD e SM maior que 5,55 e menor ou igual a 5,90. Os resultados indicando maior freqüência de DFD no músculo Semispinalis capitis e maior freqüência de PSE nos músculos Longissimus dorsi e Semimembranosus estão em acordo com Hambrecht et al. (2005) que afirmam que músculos com predominância de metabolismo oxidativo apresentam maior tendência para desenvolver DFD e os músculos com predominância de metabolismo glicolítico apresentam maior tendência para desenvolver PSE. Músculos tipo glicolíticos (por exemplo, SM e LD) produzem maior quantidade de ácido lático que os músculos tipo oxidativos (por exemplo, SC) porque usam predominantemente o mecanismo da via glicolítica para produzir energia em detrimento ao mecanismo da via oxidativa. Segundo Lengerken et al. (2002) o músculo Longissimus dorsi do suíno tem aproximadamente $13 \%$ de fibras com reação oxidativa lenta, $17 \%$ de fibras com reação oxidativa rápida e $70 \%$ de fibras com reação glicolítica rápida. Por este motivo os efeitos de manejo pré-abate que condicionam ao aparecimento da carne PSE já podem ser identificados imediatamente após o abate através do $\mathrm{pH}_{1}$ que já indica a condição de anormalidade 45 minutos após o abate. Ao contrário, em músculos com predominância de metabolismo oxidativo o DFD é uma condição melhor caracterizada através do $\mathrm{pH}_{u}$ Suínos que foram submetidos a jejum de 18 horas apresentaram maior incidência de carcaças com músculo LD e SM com $\mathrm{pH}_{\mathrm{u}}$ alto, isto é, com $\mathrm{pH}$ acima de 5,90 quando comparados em relação aos que foram submetidos a jejum de 9,12 e 15 horas. Este éum indicativo 
Tabela III. Freqüência relativa (\%) para o pH nas categorias de pH estabelecidas em função do músculo avaliado e do tempo de jejum na granja. (Relative frequency of $\mathrm{pH}_{\mathrm{u}}$ on established $\mathrm{pH}$ classes evaluated on different muscles and on farm fasting time).

\begin{tabular}{cccc}
\hline Músculo avaliado e tempo de jejum & $\mathrm{pH}_{\mathrm{u}} \leq 5,55$ & $5,55<\mathrm{pH}_{\mathrm{u}} \leq 5,90$ & $\mathrm{pH}_{\mathrm{u}}>5,90$ \\
\hline Lombo - Longissimus dorsi (LD) & $\mathrm{n}=70^{*}$ & $\mathrm{n}=120$ & $\mathrm{n}=2$ \\
9 & 31,43 & 22,03 & 0,00 \\
12 & 40,00 & 16,85 & 0,00 \\
15 & 10,00 & 33,05 & 0,00 \\
18 & 18,57 & 27,97 & 100,00 \\
Pernil - Semimembranosus (SM) & $\mathrm{n}=75$ & $\mathrm{n}=117$ & $\mathrm{n}=0$ \\
9 & 32,00 & 20,87 & 0,00 \\
12 & 34,67 & 19,13 & 0,00 \\
15 & 13,33 & 31,30 & 0,00 \\
18 & 20,00 & 28,70 & 0,00 \\
9 & $\mathrm{n}=54$ & $\mathrm{n}=130$ & $\mathrm{n}=8$ \\
Paleta - Semispinalis capitis (SC) & 38,89 & 24,39 & 12,5 \\
12 & 44,44 & 23,78 & 12,5 \\
18 & 11,11 & 26,22 & 12,5 \\
& 5,56 & 25,61 & 62,50 \\
\hline
\end{tabular}

*Freqüência absoluta em 192 avaliações realizadas para cada tipo de músculo.

que o período de 18 horas de jejum na granja tem efeito sobre as reservas de glicogênio nos músculos LD e SM de alguns suínos e condiciona para uma menor queda no $\mathrm{pH}_{u}$ em função de menor potencial de produção de ácido lático. Por outro lado, suínos que foram submetidos a jejum de 9 e 12 horas na granja apresentaram maior porcentagem de carcaças com baixo $\mathrm{pH}_{u}\left(\mathrm{pH}_{u} \leq 5,50\right)$ nos músculos LD (31,4\% e 40,0\% respectivamente) e SM (32,0\% e 34,7\%, respectivamente), enquanto que os suínos submetidos a jejum de 15 e 18 horas apresentaram maior porcentagem de carcaças com médio $\mathrm{pH}$ $\left(5,55<\mathrm{pH}_{\mathrm{u}} \leq 5,90\right)$ nos músculos LD $(33,0 \% \mathrm{e}$ $27,9 \%$, respectivamente) e SM (31,3\% e $28,7 \%$, respectivamente). Isto indica que suínos submetidos a um tempo de jejum na granja com 12 ou menos horas de duração têm reserva de glicogênio suficiente para alimentar a via glicolítica que permite a formação de ácido lático e em conseqüência é gerado um $\mathrm{pH}_{\mathrm{u}}$ mais baixo. A medida que aumenta o tempo de jejum (acima de 12 horas) a proporção de carcaças com valores intermediários de $\mathrm{pH}_{u}$ aumenta, indicando que deve ocorrer um ajuste nas rotas metabólicas para geração de energia em função da menor disponibilidade de glicogênio. Na classificação das carcaças com base no $\mathrm{pH}_{u}$ do músculo SC, observouse uma distribuição equilibrada em torno da freqüência esperada na porcentagem de carcaças (24,39\%, 23,78\%, 26,22\% e 25,61\%) com $\mathrm{pH}_{\mathrm{u}}$ bom $\left(5,55<\mathrm{pH}_{\mathrm{u}} \leq 5,90\right)$ com o incremento do período de jejum dos suínos na granja de 9, 12, 15 e 18 horas, respectivamente. O músculo SC pela característica das suas fibras musculares apresenta variação de $\mathrm{pH}$ menor e mais lenta quando comparado com os músculos LD e SM. Isto ocorre porque no processo de manutenção de homeostase celular, no período pós abate, o metabolismo oxidativo é predominante. Desta forma, devido a característica predominante dos músculos avaliados, a evolução dos valores de $\mathrm{pH}_{1}$ para $\mathrm{pH}_{u}$ apresenta um gradiente onde os as variações são menos

Archivos de zootecnia vol. 59, núm. 227, p. 400. 


\section{CONDIÇÕES PRÉ-ABATE E QUALIDADE DA CARNE DE SUÍNOS PESADOS}

intensas na paleta e maiores nos outros dois músculos e por este motivo o músculo preferencial para detectar DFD é o localizado na paleta e para detectar PSE as avaliações devem preferencialmente ser realizadas no lombo e pernil.

Em síntese: 1) A pequena freqüência de casos de DFD foi constatada na paleta de forma destacada quando o tempo de jejum na granja foi de 18 horas, 2) uma alta freqüência de casos de PSE foi constatada nos três músculos avaliados quando o tempo de jejum na granja foi de 9 ou 12 horas e, 3) adotando o tempo de jejum na granja de 15 horas é possível minimizar a freqüência de PSE no lombo e no pernil, maximizando nos dois músculos a frequëncia de carcaças com $\mathrm{pH}_{\mathrm{u}}$ na faixa adequada (maior que 5,55 e menor ou igual a 5,90$)$.

\section{BIBLIOGRAFIA}

Aaslyng, D.M. and Barton Gade, P. 2001. Low stress pre-slaughter handling: effect of lairage time on the meat quality of pork. Meat Sci., 57: 87-92.

Barton Gade, P., Christensen, L., Brown, S.N. and Warriss, P.D. 1996. Effect of tier ventilation during transport on blood parameters and meat quality in slaughter pigs. In: EU-Seminar "New Information on Welfare and Meat Quality of Pigs as Related to Handling, Transport and Lairage Conditions", 1995. Mariensee. Proceedings... Mariensee. Landbauforschung Volkenrode. pp. 101-116.

Broom, D.M., Barton Gade, P., Warriss, P., Ferlazzo, A., Hartung, J. and Manteca, X. 2002. Report of the Scientific Committee on Animal Health and Animal Welfare. The welfare of animals during transport (details for horses, pigs, sheep and cattle). European Commission. Brussels. 130 pp. Adopted on 11 March 2002.

Carr, C.C., Newman, D.J., Rentfrow, G.K., Keisler, D.H. and Berg, E.P. 2008. Effects of slaughter date, on-farm handling, transport stocking density, and time in lairage on digestive tract temperature, serum cortisol concentrations, and pork lean quality of market hogs. Prof. Anim. Sci., 24: 208-218.

\section{CONCLUSÃO}

O tempo de jejum na granja tem efeito sobre a qualidade da carne, avaliada através de $\mathrm{pH}_{1}$ e $\mathrm{pH}_{u}$, e o período de 15 horas é recomendado para ser adotado tanto no inverno quanto no verão.

Nas condições ambientais em que foi realizado o experimento não houve efeito de estação de ano e piso (inferior ou superior) da carroceria sobre o $\mathrm{pH}_{1}$ e $\mathrm{pH}_{u}$ dos músculos avaliados e sobre a perda de líquido por gotejamento no Semimembranosus.

Em condições de transporte com curta duração os suínos transportados no meio da carroceria apresentavam maiores valores de $\mathrm{pH}_{\mathrm{u}}$ nos músculos Longissimus dorsi e Semimembranosus em relação aos transportados na posição anterior e posterior da carroceria.

Chevillon, P. 1994. Le contrôle des estomacs de porcs á l'abattoir: miroir de la mise à jeun en élevage. Techni-Porc, 17: 23-30.

Christensen, L., Barton Gade, P. and Blaabjerg, L.O. 1994. Investigation of transport conditions in participating countries in the EC Project: PL 920262. Proceedings of $40^{\text {th }}$ International Congress of Meat Science and Technology. The Hague, NL. Paper W-2.01.

Dalla Costa, O.A., Ludke, J.V., Paranhos da Costa, M.J.R., Faucitano, L., Peloso, J.V. and Dalla Roza, D. 2007a. Impact of the truck design on welfare and meat quality in pig. Ciênc. Rural, 37: 1418-1422.

Dalla Costa, O.A., Faucitano, L., Coldebella, A., Ludke, J.V., Peloso, J.V., Dalla Rosa, D. and Paranhos da Costa, M.J.R. 2007b. Effects of the season of the year, truck type and location on truck on skin bruises and meat quality in pigs. Livest. Prod. Sci., 107: 29-36.

Dalla Costa, O.A., Ludke, J.V., Paranhos da Costa, M.J.R., Faucitano, L., Coldebella, A., Kich, J.D., Peloso, J.V. and Dalla Roza, D. 2008a. Fasting time at farm on hormonal status and physiological parameters of heavy-weight slaughter pigs. Ciênc. Rural, 38: 2300-2308.

Dalla Costa, O.A., Paranhos da Costa, M.J.R.,

Archivos de zootecnia vol. 59, núm. 227, p. 401. 


\section{DALLA COSTA, LUDKE, COSTA, FAUCITANO, PELOSO E DALLA ROZA}

Ludke, J.V., Coldebella, A., Kich, J.D., Peloso, J.V., Faucitano, L. and Dalla Roza, D. 2008b. Relation of fasting time during pre-slaughter management to weight loss, weight of stomach contents and incidence of gastric ulcer in pigs. Ciênc. Rural, 38: 199-205.

Eikelenboon, G., Bolink, A.H. and Sybesma, E. 1991. Effects of feed withdrawal before delivery on pork quality and carcass yield. Meat Sci., 29: 25-30.

Faucitano, L. 2007. Preslaughter stressors effects on pork: a review. J. Muscle Foods, 9: 293-303.

Guardia, M.D., Gispert, M. and Diestre, A. 1996. Mortality rates during transport and lairage in pigs for slaughter. Meat Foccus Internat., 4: 362-366.

Hambrecht, E. 2004. Critical pre and postslaughter factors em relation to pork quality. Thesis (Doctor). Wageningen Institute of Animal Sciences. Wageningen University. Wageningen. 151 pp.

Hambrecht, E., Eissen, J.J., Newmann, D.J., Smits, C.H.M., Verstegen, M.W.A. and Den Hartog, L.A. 2005. Preslaughter handling effects on pork quality and glycolytic potencial in two muscles differing in fiber type composition. $J$. Anim. Sci., 89: 900-907.

Honikel, K.O. 1998. Reference methods for the assessment of physical characteristics of meat Meat Sci., 49: 447-457.

Jones, S.D.M., Rompala, R.E. and Haworth, C.R. 1985. Effects of fasting and water restriction on carcass shrink and pork quality. Can. J. Anim. Sci., 65: 613-619.

Lengerken, G., Steffen, M. and Wicke, M. 2002. Muscle metabolism and meat quality of pigs and poultry. Veternarija ir Zootechnika T., 20: 8286.

Magras, C., Delaunay, I. et Bénéteau, E. 2000. Quelles durées de mise à jeun des porcs charcutiers pour um optimun de qualité dês carcasses? Détermination à partir dún étude terrain. Journées Recherche Porcine em France, 32: 351-356.

Murray, A.C. 2000. Reducing losses from farm gate to packer. Adv. Pork Produc., 11: 175- 180.

Murray, C. and Jones, S.D.M. 1994. The effect of mixing, feed restriction and genotype with respect to stress susceptibility on pork carcass and meat quality. Can. J. Anim. Sci., 74: 587-594.

Murray, C., Robertson, W., Nattress, F. and Fortin, A. 2001. Effect of preslaughter overnight feed withdrawal on pig carcass and muscle quality. Can. J. Anim. Sci., 81: 89-97.

Otto, G., Roehe, R., Looft, H., Thoelking, L. and Kalm, E. 2004. Comparision of different methods for determination of drip loss and their relationships to meat quality and carcass characteristics. Meat Sci., 68: 401-409.

Ryan, M.H. 2007. Influence of seasonal environment, top and botton deck transport, transport duration, and time in lairage on overall pork quality and blood serum cortisol concentrations of market hogs. Thesis (Master of Science). University of Missouri. Columbia. 55 pp.

SCAHAW. 1999. Scientific Committee on Animal Health and Animal Welfare. Standards for the Microclimate inside Animal Transport Road Vehicles. $8^{\text {th }}$ Decembeer of 1999. Sanco/B3/ AW/R13/1999. Disponible at http://europa.eu.int/ comm/food/fs/sc/scah/out35_en.html

SAS. 1999-2001. System for Microsoft Windows, Release 8.2. Cary. (cd-rom).

Smet, S.M., Pauwels, H., Bie, S., Demeyer, D.L., Callewier, J. and Eeckhount, W. 1996. Effects of halothane genotype breed feed withdrawal and lairage on pork quality of Belgian slaughter pigs. J. Anim. Sci., 74: 1854-1863.

Wittmann, W., Ecolan, P., Levasseur, P. and Fernandez, X. 1994. Fasting-Induced glycogen depletion in different fibre types of red and white pig muscles - Relationship with ultimate pH. J. Food Agric., 66: 257-266.

Archivos de zootecnia vol. 59, núm. 227, p. 402. 